Revue Française de Gestion Industrielle

Vol. 20, $\mathrm{N}^{\circ} 3$

\title{
ANALYSE D'OUVRAGE
}

\author{
Hugues Molet \\ A propos du livre de Philippe NIEWBOURG et Hubert D'HONDT \\ PLACE DE MARCHE SUR INTERNET \\ NOUVELLES REGLES POUR LE COMMERCE DU $21^{\text {IEME }}$ SIECLE
}

Editions BNTP - 2001

J'ai été attiré naturellement par le titre de cet ouvrage, et je dois dire que je n'ai pas du tout regretté sa lecture; je la conseille, c'est un ouvrage facile à lire, intéressant ; les auteurs croient à Internet, aux places de marché, à leur développement, et s'ils cherchent à faire participer leur thèse, ils gardent toujours une certaine prudence. L'ouvrage adopte un ton familier qui rend sa lecture agréable.

Son objectif est défini dès le début : «essayons d'y voir plus clair dans la jungle de ce qu'il est convenu d'appeler la nouvelle économie et les places de marché et d'esquisser les évolutions possibles ».

D'abord un rappel important : Internet et le commerce électronique ne sont pas vraiment une révolution de concepts; ce qui est révolutionnaire, ce sont les possibilités nouvelles qu'offrent la vitesse et la quantité d'informations susceptibles d'être transmises à n'importe quel coin de la planète.

Les auteurs commencent par dresser une fresque des évolutions des moyens de transport, des modes de commercialisation et de transmission de l'information depuis des siècles. Ils montrent tout a permis d'étendre les réseaux et leurs conséquences médiatiques; nous en sommes aujourd'hui à une capacité de transmission de près d'un million de caractères par seconde n'importe où dans le monde. Ceci a un impact gigantesque sur les prix, la concurrence mondiale, l'harmonisation des législations mondiales, impact dont on ne voit d'ailleurs pas 
encore toutes les conséquences. Une étude du Gartner Group évalue la multiplication du chiffre d'affaires des entreprises européennes par un facteur 70 avant 2004 !

Internet et le commerce électronique vont donc remettre profondément en cause les circuits de distribution classique par le phénomène de «desintermédiation ». Certaines entreprises sont encore réfractaires (les brick and mortar), d'autres tentent certaines incursions dans ce nouveau monde par une distribution mixte (les click and mortar), enfin les dernières n'ont bâti leur modèle entrepreneurial que sur ce modèle (les dot corn), les stratégies des unes et des autres dépendant évidemment de leur taille et de leurs types de marché.

Les entreprises peuvent distribuer désormais directement leur information sans intermédiaire; certaines ne vendent d'ailleurs que de l'information: par exemple, celles qui ne servent que d'intermédiaires entre vendeurs et acheteurs, «les infomédiaires ». Nous voici entrés dans le monde des places de marché.

Une première comparaison intéressante des places de marché électroniques (PdM) est réalisée avec la place du marché de village où l'on trouve des acteurs et des caractéristiques analogues à ceux des PdM : vendeurs, acheteurs, lieu, transactions, promotion, marketing et même organisateur : le placier!

La PdM fonctionne d'une manière analogue, mais c'est un peu plus compliqué : on trouve le catalogue des produits et services, 1' identification des acheteurs, un organisateur qui va réaliser, grâce à un moteur de recherche, les transactions et qui se paiera sur celles-ci. Ces transactions sont enregistrées, des dialogues techniques peuvent même être organisés; reste le paiement qui demeure un peu en retard dans le développement, les cartes électroniques sociétés et la signature électronique restant encore peu opérationnelles, du moins en France.

Ces nouvelles potentialités vont modifier totalement la concurrence puisque désormais l'information est disponible pour tous et partout; le pouvoir réside dans la détention de l'information. Mais qui dit information partagée et disponible dit risques tant du côté du vendeur que de celui de l'acheteur et se pose évidemment le problème du choix de la PdM la mieux adaptée à vos besoins, voire de l'opportunité de sa création.

Les auteurs vont alors dresser des critères de typologie des places de marché existantes.

Premier critère : le nombre d'acteurs.

Supposons que nous ayons $\mathrm{N}$ vendeurs et $\mathrm{M}$ acheteurs.

$\mathrm{N}=1$ : ce n'est que du commerce électronique

$\mathrm{N}=\mathbf{1} \mathrm{M}=\mathbf{1}$ : c'est de l'extranet basé sur des systèmes EDI

$M=1$; c'est une centrale d'achats ou un portail basé sur Internet: on centralise les achats pour bénéficier d'un effet de taille. 
Avec $\mathrm{N}$ et $\mathrm{M}$ quelconques, on a vraiment une PdM complète.

On peut utiliser d'autres critères de typologie comme par exemple le type d'achats :

- achats stratégiques, ceux de matières premières ou de machines

- dans ce cas, des économies de 'massification' sont possibles grâce aux plate-formes d'achats qui permettent de standardiser les appels d'offre

- achats hors production (fournitures ou services) ou encore achats

- de fournitures tout secteur ou toutes fournitures pour un secteur.

- Dans ces deux cas, sont générés des coûts administratifs pouvant être gérés par des work-flow.

Une autre typologie intéressante est celle des niveaux de communication :

- Niveau 1 : simple mise en relation et saisie de l'information de base

- Niveau 2: l'appel d'offres; ce lui-ci est formaté pour que les fournisseurs puissent répondre directement; la $\operatorname{PdM}$ ne joue qu'un rôle d'intermédiation acheteursvendeurs.

- Niveau 3: le commerce en ligne; il y a désormais un «méta-catalogue » de produits harmonisés ; la transaction a lieu sur site ou à l'extérieur de façon classique.

- Niveau 4: le paiement se fait désormais en ligne; il ne concerne que les petits montants (grand public); le paiement constitue un maillon faible du commerce électronique à cause des problèmes juridiques et de sécurité.

- Niveau 5 : intégration du service client; on fournit des services complémentaires au client: suivi de ses stocks, gestion des approvisionnements, recherche des meilleurs fournisseurs, mutualisation de services...

- Niveau 6: intégration avec la totalité de la ligne logistique; les systèmes d'information clients et fournisseurs sont mis en relation, par exemple, si l'on détecte une rupture de stock chez un client, un fournisseur est automatiquement consulté.

- Niveau 7 : le design collaboratif ; la commande est personnalisée via la PdM (exemple des ordinateurs DELL); l'utilisation de bibliothèques de données techniques devrait même permettre des projets de travail commun en conception (CAO par Internet)

Abordons maintenant les critères financiers; la dernière typologie propose de distinguer les modèles économiques suivants : 
- le modèle gratuit : financement par la publicité et la vente de fichiers

- le modèle acteurs entièrement financé par les acheteurs qui mutualisent leurs achats et leurs services

- le modèle communautaire financé par abonnement fixe, quelle que soit l'utilisation

- le modèle poker : on a accès à l'appel d'offres mais le contact avec le vendeur est facturé

- le modèle commission; on touche une commission sur la transaction (généralement payée par le vendeur)

- les modèles mixtes, qui combinent les procédures des différents modèles précédents.

Quels sont les outils qui permettent le fonctionnement de ces différents modèles en assurant traçabilité, respect juridique, protection des clients et fournisseurs...

Ces outils sont désormais classiques; ce qui caractérise la PdM, c'est leur utilisation conjointe et interfacée. Donnons quelques exemples :

- le méta-catalogue des différents fournisseurs; à ce niveau, le problème de fond reste celui de la standardisation des langages (le plus récent est : XML, extended Markup Langage)

- le work-flow pour suivre la gestion de toutes les étapes des transactions selon des procédures définies et paramétrées

- les outils de gestion des profils, qui permettent de mettre en relation clients et fournisseurs

- les outils de back office, c'est-à-dire ceux qui permettent la gestion interne de la PdM, par exemple: les facturations, le data-warehouse ou encore le data-mining, c'est à dire la recherche d'informations-clés ou de tendances dans l'ensemble du datawarehouse.

Les auteurs abordent ensuite d'autres aspects, comme par exemple les coûts des PdM. Ceux-ci dépendent naturellement du type de prestations, du nombre d'acteurs, de produits, de l'audience, du type d'outils... Les coûts s 'échelonnent de 2 à plusieurs millions de francs, les coûts difficiles à évaluer sont naturellement ceux de maintenance et de développement ainsi que les interfaçages avec les systèmes clients et fournisseurs.

Un autre aspect intéressant abordé en fin d'ouvrage est celui des innovations que les PdM permettent d'inclure dans la relation client: il s'agit des outils CRM (Customer Relationship Management). Ces outils possèdent de nombreuses fonctionnalités, comme par exemple: les campagnes marketing personnalisées (One to One), l'historique des relations permettant des 
suivis d'actions commerciales; la performances de ces outils dépend fortement des capacités d'interfaçages avec systèmes clients et fournisseurs.

L'ouvrage se termine par les perspectives d'évolution. Les auteurs reconnaissent que la rapidité actuelle de développement rend toute prévision hasardeuse, mais ils prévoient quelques tendances :

- la centralisation sur quelques grosses PdM

- le développement de connections sans fil qui assurera une nouvelle portabilité

- le développement avec des applications Internet pour assurer encore davantage de relations BtoBtoC, comme dans exemple de l'employé gérant directement ses congés, sa formation (Business to Employee)

- l'ingénierie collective ou l'usine numérique où tous collaborent dans une chaîne logistique numérique incluant même les fonctions de conception partagée. Les auteurs reconnaissent qu'à ce niveau l'un des maillons faibles reste celui de la logistique physique, celle du dernier kilomètre .

On trouve à la fin un glossaire de la terminologie PdM, des adresses de sites PdM, et celles des principaux créateurs de logiciels.

Un livre bien intéressant, d'actualité, de lecture facile et agréable ; on lui pardonnera bien volontiers quelques redondances et une absence de présentation d'échecs qui auraient pu parfois nuancer l'enthousiasme sympathique des auteurs. 